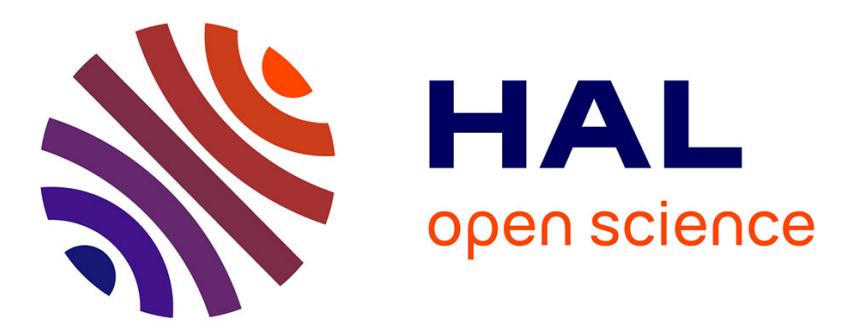

\title{
SOME REMARKS ON STRONG APPROXIMATION AND APPLICATIONS TO HOMOGENEOUS SPACES OF LINEAR ALGEBRAIC GROUPS
}

F Balestrieri

\section{- To cite this version:}

F Balestrieri. SOME REMARKS ON STRONG APPROXIMATION AND APPLICATIONS TO HOMOGENEOUS SPACES OF LINEAR ALGEBRAIC GROUPS. 2020. hal-03013498

\author{
HAL Id: hal-03013498 \\ https://hal.science/hal-03013498
}

Preprint submitted on 19 Nov 2020

HAL is a multi-disciplinary open access archive for the deposit and dissemination of scientific research documents, whether they are published or not. The documents may come from teaching and research institutions in France or abroad, or from public or private research centers.
L'archive ouverte pluridisciplinaire HAL, est destinée au dépôt et à la diffusion de documents scientifiques de niveau recherche, publiés ou non, émanant des établissements d'enseignement et de recherche français ou étrangers, des laboratoires publics ou privés. 


\title{
SOME REMARKS ON STRONG APPROXIMATION AND APPLICATIONS TO HOMOGENEOUS SPACES OF LINEAR ALGEBRAIC GROUPS
}

\author{
F. BALESTRIERI
}

\begin{abstract}
Let $k$ be a number field and $X$ a smooth, geometrically integral quasi-projective variety over $k$. For any linear algebraic group $G$ over $k$ and any $G$-torsor $g: Z \rightarrow X$, we observe that if the étale-Brauer obstruction is the only one for strong approximation off a finite set of places $S$ for all twists of $Z$ by elements in $H_{\text {ét }}^{1}(k, G)$, then the étale-Brauer obstruction is the only one for strong approximation off a finite set of places $S$ for $X$. As an application, we show that any homogeneous space of the form $G / H$ with $G$ a connected linear algebraic group over $k$ satisfies strong approximation off the infinite places with étale-Brauer obstruction, under some compactness assumptions when $k$ is totally real. We also prove more refined strong approximation results for homogeneous spaces of the form $G / H$ with $G$ semisimple simply connected and $H$ finite, using the theory of torsors and descent.
\end{abstract}

\section{INTRODUCTION AND MAIN RESULTS}

Motivation. The aim of this short note is to collect together various observations on strong approximation for points on varieties over number fields, with a view towards applications to homogeneous spaces of connected linear algebraic groups. In particular, in Theorem 1.5 we will show that, if $k$ is a number field, $G$ is a connected linear algebraic group over $k, H \subset G$ is any closed $k$-subgroup (not necessarily normal nor connected), and $S$ is any finite set of places containing all the infinite places such that some non-compactness conditions for the adelic points at $S$ of the semisimple part of $G$ hold, then the homogeneous space $G / H$ satisfies strong approximation with étale-Brauer obstruction off $S$. (A similar result has been recently found independently by Demeio in [Dem20]; see also Remark 1.6.) In the special case when $G$ is semisimple simply connected (e.g. $\mathrm{SL}_{n, k}$ ) and $H$ is finite, we will give even more precise statements for strong approximation, using the theory of torsors and descent (see Theorem 1.10 below). This approach using torsors complements the one trying to obtain information about strong approximation using the Brauer-Manin obstruction (e.g. [BD13, Dem17, [CX18a, ,CX18b], Cao18], [CDX19]). Homogeneous spaces of the form $\mathrm{SL}_{n, k} / H$ with $H$ finite and constant are of particular interest for their connection to the inverse Galois problem: if $\mathrm{SL}_{n, k} / H$ satisfies strong approximation off some finite set $S$, then it satisfies weak weak approximation (that is, weak approximation away from some finite set of places), and thus, by [Ser08, §3] and [Eke90], the inverse Galois problem for $H$ has a positive answer. (In fact, in Har07 Harari showed that an even weaker property than weak weak approximation, called hyper-weak approximation, suffices to guarantee that the inverse Galois problem for $H$ holds.)

Obstruction sets and strong approximation. Let us now give more specific details about strong approximation and the types of obstruction sets that we are going to use. Let $k$ be a number field with a fixed algebraic closure $\bar{k}$ and let $\mathbf{A}_{k}$ the adelic ring of $k$. Let $\Omega_{k}$ be the set of non-trivial places of $k$ and $\infty_{k} \subset \Omega_{k}$ be the subset of infinite places. For each place $v \in \Omega_{k}$, we write $k_{v}$ for the completion of $k$ at $v$. Let $X$ a smooth, geometrically integral, quasi-projective variety over $k$, where by "variety over $k$ " we mean a separated scheme of finite type over $k$. We write $\bar{X}:=X \times_{\operatorname{Spec} k} \operatorname{Spec} \bar{k}$. We now define some useful subsets of the set of adelic points $X\left(\mathbf{A}_{k}\right)$, all containing the adelic closure of $X(k)$.

Date: June 16, 2020. 
Let $\operatorname{Br} X:=H_{\text {ét }}^{2}\left(X, \mathbf{G}_{m}\right)$ be the (cohomological) Brauer group of $X$. Under our assumptions on $X$, it follows by a theorem of Gabber that $\operatorname{Br} X$ coincides with the Brauer group defined via Azumaya algebras (see $\mathrm{dJ}]$ ).

Definition 1.1. For any subset $B \subset \operatorname{Br} X$, we define the set

$$
X\left(\mathbf{A}_{k}\right)^{B}:=\bigcap_{\alpha \in B}\left\{\left(x_{v}\right) \in X\left(\mathbf{A}_{k}\right): \sum_{v \in \Omega_{k}} \operatorname{inv}_{v}\left(\alpha\left(x_{v}\right)\right)=0\right\},
$$

where the maps $\operatorname{inv}_{v}: \operatorname{Br} k_{v} \rightarrow \mathbf{Q} / \mathbf{Z}$ are the invariant maps coming from local class field theory. When $B=\operatorname{Br} X$ (respectively, $B=\operatorname{Br}_{1} X:=\operatorname{ker}(\operatorname{Br} X \rightarrow \operatorname{Br} \bar{X})$ ), we call $X\left(\mathbf{A}_{k}\right)^{B}$ the Brauer-Manin set (respectively, the algebraic Brauer-Manin set) of $X$.

Recall that, for any linear algebraic group $G$ over $k$, the cohomology set $H_{\text {ét }}^{1}(X, G)$ classify $(X$ isomorphism) classes of $G$-torsors $g: Z \rightarrow X$. Moreover, for any 1-cocycle $\sigma \in Z_{\text {ét }}^{1}(k, G)$, we can twist any $G$-torsor $g: Z \rightarrow X$ by $\sigma$ to obtain a $G^{\sigma}$-torsor $g^{\sigma}: Z^{\sigma} \rightarrow X$ (see e.g. [Sko01] for more details). Since the isomorphism classes of $G^{\sigma}$ and of the $G^{\sigma}$-torsor $g^{\sigma}: Z^{\sigma} \rightarrow X$ depend on the class $[\sigma] \in H_{\text {ét }}^{1}(k, G)$ only, we will abuse notation and refer to twisting $G$-torsors $g: Z \rightarrow X$ by $\sigma \in H_{\text {ét }}^{1}(k, G)$, writing $g^{\sigma}: Z^{\sigma} \rightarrow X$ for the twisted $G^{\sigma}$-torsor.

Let $\mathcal{F}_{k}$ (respectively, $\mathcal{F}_{k}^{\text {ab }}, \mathcal{F}_{k}^{\text {nilp }}, \mathcal{F}_{k}^{\text {sol }}$ ) be the set of all $k$-isomorphism classes of finite (respectively, finite abelian, finite nilpotent, finite solvable) linear algebraic groups over $k$.

Definition 1.2. For $\star \in\left\{\mathcal{F}_{k}, \mathcal{F}_{k}^{\text {ab }}, \mathcal{F}_{k}^{\text {nilp }}, \mathcal{F}_{k}^{\text {sol }}\right\}$ and $\square \in\{, \operatorname{Br}\}$, consider the set

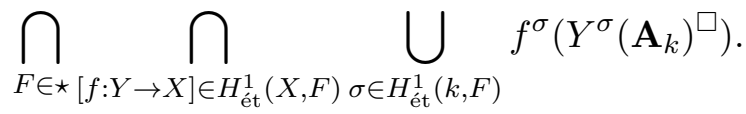

We call such a set the

- finite descent set of $X$, denoted by $X\left(\mathbf{A}_{k}\right)^{\text {ét }}$, if $(\star, \square)=\left(\mathcal{F}_{k},\right)$;

- finite abelian descent set of $X$, denoted by $X\left(\mathbf{A}_{k}\right)^{\mathrm{f}-\mathrm{ab}}$, if $(\star, \square)=\left(\mathcal{F}_{k}^{\mathrm{ab}},\right)$;

- finite nilpotent descent set of $X$, denoted by $X\left(\mathbf{A}_{k}\right)^{\mathrm{f}-\text {-nilp }}$, if $(\star, \square)=\left(\mathcal{F}_{k}^{\text {nilp }},\right)$;

- finite solvable descent set of $X$, denoted by $X\left(\mathbf{A}_{k}\right)^{\text {f-sol }}$, if $(\star, \square)=\left(\mathcal{F}_{k}^{\text {sol }},\right)$;

- étale-Brauer set of $X$, denoted by $X\left(\mathbf{A}_{k}\right)^{\text {ét Br }}$, if $(\star, \square)=\left(\mathcal{F}_{k}, \mathrm{Br}\right)$.

If $S \subset \Omega_{k}$ is a finite subset of places of $k$, we let

$$
\operatorname{pr}^{S}: X\left(\mathbf{A}_{k}\right) \rightarrow X\left(\mathbf{A}_{k}^{S}\right):=\prod_{v \notin S}^{\prime}\left(X\left(k_{v}\right), \mathcal{X}\left(\mathcal{O}_{v}\right)\right)
$$

be the natural projection, where $\mathcal{X}$ is some smooth and separated model of $X$ over some open subset of $\operatorname{Spec}\left(\mathcal{O}_{k}\right)$, and where $\Pi^{\prime}$ denotes the restricted product (as convention, when $v \in \infty_{k}$ we take $X\left(k_{v}\right)$ in the restricted product). The map $\operatorname{pr}^{S}$ is surjective if $\prod_{v \in S} X\left(k_{v}\right) \neq \emptyset$. If $\omega$ is some "obstruction", we let $X\left(\mathbf{A}_{k}^{S}\right)^{\omega}:=\operatorname{pr}^{S}\left(X\left(\mathbf{A}_{k}\right)^{\omega}\right)$. We denote by $\delta: X(k) \rightarrow X\left(\mathbf{A}_{k}\right)$ the natural diagonal map. To ease notation, we denote the closure of $\operatorname{pr}^{S}\left(\delta(X(k))\right.$ in $X\left(\mathbf{A}_{k}^{S}\right)$ by $\overline{X(k)}^{S}$.

Definition 1.3. Let $X$ be a geometrically integral variety over a number field $k$. Let $S \subset \Omega_{k}$ be a finite (possibly empty) subset of places of $k$. Let $\omega$ be some "obstruction" and suppose that $X\left(\mathbf{A}_{k}\right)^{\omega} \neq \emptyset$. We say that $X$ satisfies strong approximation off $S$ with obstruction $\omega$ if $\operatorname{pr}^{S}(\delta(X(k)))$ is dense in $X\left(\mathbf{A}_{k}^{S}\right)^{\omega} \subset X\left(\mathbf{A}_{k}^{S}\right)$ with respect to the subspace topology induced by the topology on $X\left(\mathbf{A}_{k}^{S}\right)$ or, equivalently, if $X\left(\mathbf{A}_{k}^{S}\right)^{\omega} \subset \overline{X(k)}^{S}$ in $X\left(\mathbf{A}_{k}^{S}\right)$.

Main results. We can now state the main results of this short note. We start with the following observation, which is a corollary of [Cao20, Théorème 1.1]. 
Theorem 1.4. Let $X$ be a smooth and geometrically integral quasi-projective variety over a number field $k$ such that $X\left(\mathbf{A}_{k}\right)^{\text {ét } \mathrm{Br}} \neq \emptyset$. Let $G$ be a linear algebraic group over $k$ and let $g: Z \rightarrow X$ be $a$ $G$-torsor. Let $S \subset \Omega_{k}$ be a finite (possibly empty) subset of places of $k$. Suppose that $Z^{\sigma}\left(\mathbf{A}_{k}^{S}\right)^{\text {ét } \mathrm{Br}} \subset$ ${\overline{Z^{\sigma}(k)}}^{S}$ for all $\sigma \in H_{\text {ét }}^{1}(k, G)$ such that $Z^{\sigma}\left(\mathbf{A}_{k}\right)^{\text {ét } \mathrm{Br}} \neq \emptyset$, that is, that the étale-Brauer obstruction is the only one for strong approximation off $S$ for all $Z^{\sigma}$. Then $X\left(\mathbf{A}_{k}^{S}\right)^{\text {ét } \mathrm{Br}} \subset \overline{X(k)}$, that is, the étale-Brauer obstruction is the only one for strong approximation off $S$ for $X$.

Theorem 1.4, together with some recent results on strong approximation for connected linear algebraic groups, yields the following quite general application to homogeneous spaces of connected linear algebraic groups.

Theorem 1.5. Let $G$ be a connected linear algebraic group over a number field $k$ and let $H \subset G$ be a closed (not necessarily normal nor connected) k-subsgroup of $G$. Let $S \subset \Omega_{k}$ be a finite subset of places of $k$ with $\infty_{k} \subset S$ such that the following non-compactness condition holds: viewing $G \rightarrow G / H$ as an $H$-torsor, for any $\sigma \in H_{\text {ét }}^{1}(k, H)$ we have that $\prod_{v \in S} G_{\sigma}^{\prime}\left(k_{v}\right)$ is not compact for each non-trivial $k$-almost simple factor $G_{\sigma}^{\prime}$ of the semisimple part of $G^{\sigma}$. Then $G / H$ satisfies strong approximation off $S$ with étale-Brauer obstruction.

Remark 1.6. A similar result as Theorem 1.5 has been recently proved, independently and with different techniques, by Julian Demeio (see Dem20, Theorem 5.0.1]). In his work, Demeio then obtains further nice results by considering the set $X\left(\mathbf{A}_{k}^{S}\right)^{\omega_{S}}$ (i.e. the set of points in $X\left(\mathbf{A}_{k}^{S}\right)$ which survive the obstruction $\omega_{S}$ given by, roughly speaking, the "obstruction $\omega$ with the places $S$ removed"). In this paper, we go on a different direction, looking instead at descent obstructions for homogeneous spaces of the form $G / H$, where $G$ is semisimple simply connected and $H$ is finite.

Remark 1.7. If $k$ is not totally real, then the non-compactness conditions in Theorem 1.5 are vacuous.

Remark 1.8. If $\bar{k}[G]^{\times}=\bar{k}^{\times}$, then by the main theorem in Cao18 we can take $S \subset \Omega_{k}$ to be any non-empty finite subset (not necessarily containing $\infty_{k}$ ) in Theorem 1.5 .

Remark 1.9. There are examples of homogeneous spaces of connected linear algebraic groups for which strong approximation with Brauer-Manin obstruction is not the only one (see e.g. Dem17] for an example of such a homogeneous space $G / H$ with $G$ semisimple simply connected and $H$ finite nilpotent).

If we specialise to the case when $G$ is a semisimple simply connected linear algebraic group over $k$ (e.g. $G=\mathrm{SL}_{n, k}$ for $n \geq 2$ ) and $H \subset G$ is a finite closed $k$-subgroup, we can obtain more precise results on strong approximation, using the theory of torsors. Recall that, if $\mathscr{F}$ is a subset of $(k$-isomorphism classes of) finite linear algebraic groups over $k$ and if $X$ is a variety over $k$, then

$$
X\left(\mathbf{A}_{k}\right)^{\mathscr{F}}:=\bigcap_{F \in \mathscr{F}} \bigcap_{[f: Y \rightarrow X] \in H_{\mathrm{et}}^{1}(X, F)} \bigcup_{\sigma \in H_{\mathrm{et}}^{1}(k, F)} f^{\sigma}\left(Y^{\sigma}\left(\mathbf{A}_{k}\right)\right) .
$$

Theorem 1.10. Let $k$ be a number field and let $\mathscr{F} \subset \mathcal{F}_{k}$ be a set of ( $k$-isomorphism classes of) finite linear algebraic groups over $k$ which is geometrically closed under taking quotients in the sense that if $F \in \mathcal{F}_{k}$ is such that there exist $F_{1} \in \mathscr{F}$ and $F_{2} \in \mathcal{F}_{\bar{k}}$ satisfying $F(\bar{k})=F_{1}(\bar{k}) / F_{2}(\bar{k})$, then $F \in \mathscr{F}$. Let $G$ be a semisimple simply connected linear algebraic group over $k$ and let $H \subset G$ be any finite closed $k$-subgroup with $H \in \mathscr{F}$. Let $S \subset \Omega_{k}$ be a finite non-empty subset of places of $k$ such that $\prod_{v \in S} G_{\sigma}^{\prime}\left(k_{v}\right)$ is not compact for each non-trivial $k$-almost simple factor $G_{\sigma}^{\prime}$ of $G^{\sigma}$, for any $\sigma \in H_{\text {ét }}^{1}(k, H)$. Then $(G / H)$ satisfies strong approximation off $S$ with the descent obstruction associated to $\mathscr{F}$, that is, $(G / H)\left(\mathbf{A}_{k}^{S}\right)^{\mathscr{F}} \subset \overline{(G / H)(k)}^{S}$.

Example 1.11. Feasible (in the sense of Theorem 1.10 choices for $\mathscr{F}$ include: all \{finite, finite abelian, finite solvable, finite supersolvable, finite nilpotent\} linear algebraic groups over $k$. When 
$\mathscr{F}=\mathcal{F}_{k}^{\mathrm{ab}}$, then $(G / H)\left(\mathbf{A}_{k}^{S}\right)^{\mathrm{f}-\mathrm{ab}} \subset \overline{(G / H)(k)}^{S}$. Since $\bar{k}[G / H]^{\times}=\bar{k}^{\times}$, by Har02, Théorème 2] we have $(G / H)\left(\mathbf{A}_{k}\right)^{\mathrm{Br}_{1}} \subset(G / H)\left(\mathbf{A}_{k}\right)^{\mathrm{f}-\mathrm{ab}}$, so that $(G / H)\left(\mathbf{A}_{k}^{S}\right)^{\mathrm{Br}{ }_{1}} \subset \overline{(G / H)(k)}{ }^{S}$ as well; we compare this with [Bor96 for a similar result concerning weak approximation with algebraic Brauer-Manin obstruction. When $\mathscr{F}=\mathcal{F}_{k}^{\text {sol }}$, we refer to HW18 for results concerning weak approximation with Brauer-Manin obstruction in the case when $H$ is supersolvable (in the sense of [HW18, Définition 6.4]), and hence solvable. It is unclear to the author what the relation between $(G / H)\left(\mathbf{A}_{k}^{S}\right)^{\mathrm{Br}}$ and $(G / H)\left(\mathbf{A}_{k}^{S}\right)^{\mathrm{f} \text {-sol }}$ is. When $\mathscr{F}=\mathcal{F}_{k}^{\text {nilp}}$, we know by Dem17] that there are examples of number fields $k$, finite non-empty subsets $S \subset \Omega_{k}$ of places of $k$, and homogeneous spaces $G / H$ with $G$ semisimple simply connected and $H$ finite nilpotent such that strong approximation off $S$ with Brauer-Manin obstrution fails, in the sense that $(G / H)\left(\mathbf{A}_{k}^{S}\right)^{\mathrm{Br}}$ is not contained in $\overline{(G / H)(k)}{ }^{S}$. Explicitly, one can take, for example, $k=\mathbf{Q}(\sqrt{-21}), S=\infty_{k} \cup\{v \mid 2\}, G=\mathrm{SL}_{4, k}$, and $H=Q_{8}$ (the quaternion group of order 8). By our Theorem 1.10, these counterexamples by Demarche are all explained by the finite nilpotent descent obstruction and they show, in particular, that $(G / H)\left(\mathbf{A}_{k}^{S}\right)^{\mathrm{Br}} \not \subset(G / H)\left(\mathbf{A}_{k}^{S}\right)^{\mathcal{F}_{k}^{\text {nilp }}}$ in such cases.

Remark 1.12. In the light of Theorem 1.10, it would be interesting to study further the relationships (if any) between descent sets under classes of finite linear algebraic groups and Brauer-Manin sets.

\section{ProOFS}

We now give proofs of the results presented in the previous section.

Proof of Theorem 1.4. Since by Cao20, Théorème 1.1] we have $X\left(\mathbf{A}_{k}\right)^{\text {ét } \mathrm{Br}}=\bigcup_{\sigma} g^{\sigma}\left(Z^{\sigma}\left(\mathbf{A}_{k}\right)^{\text {ét Br}}\right)$, it follows that $X\left(\mathbf{A}_{k}^{S}\right)^{\text {ét Br }}=\operatorname{pr}^{S}\left(\bigcup_{\sigma} g^{\sigma}\left(Z^{\sigma}\left(\mathbf{A}_{k}\right)^{\text {ét Br}}\right)\right)=\bigcup_{\sigma} g^{\sigma}\left(Z^{\sigma}\left(\mathbf{A}_{k}^{S}\right)^{\text {ét Br }}\right)$. By our assumptions, we deduce that

$$
\begin{aligned}
X\left(\mathbf{A}_{k}^{S}\right)^{\text {ét Br }} & =\bigcup_{\sigma} g^{\sigma}\left(Z^{\sigma}\left(\mathbf{A}_{k}^{S}\right)^{\text {ét Br }}\right) \\
& =\bigcup_{\sigma} g^{\sigma}\left(\overline{Z^{\sigma}(k)} \cap Z^{\sigma}\left(\mathbf{A}_{k}^{S}\right)^{\text {ét Br }}\right) \\
& \subset \bigcup_{\sigma}\left(g^{\sigma}\left(\overline{Z^{\sigma}(k)}\right)^{S} \cap g^{\sigma}\left(Z^{\sigma}\left(\mathbf{A}_{k}^{S}\right)^{\text {ét Br }}\right)\right) \\
& \left.\subset\left(\bigcup_{\sigma} g^{\sigma}\left(\overline{Z^{\sigma}(k)}\right)^{S}\right)\right) \cap\left(\bigcup_{\sigma} g^{\sigma}\left(Z^{\sigma}\left(\mathbf{A}_{k}^{S}\right)^{\text {ét Br }}\right)\right) \\
& \subset \overline{X(k)} S \cap X\left(\mathbf{A}_{k}^{S}\right)^{\text {é Br }} \\
& \subset \overline{X(k)} S
\end{aligned}
$$

where in the second-to-last inclusion we have used that $g^{\sigma}\left({\overline{Z^{\sigma}(k)}}^{S}\right) \subset \overline{X(k)}^{S}$ for all $\sigma \in H_{\text {ét }}^{1}(k, G)$. We quickly sketch a proof of this fact. Assume that $Z^{\sigma}(k) \neq \emptyset$, otherwise there is nothing to prove. Let $\left(z_{v}\right) \in{\overline{Z^{\sigma}(k)}}^{S}$ and consider $\left(x_{v}\right):=g^{\sigma}\left(\left(z_{v}\right)\right) \in X\left(\mathbf{A}_{k}^{S}\right)$. Let $S_{0} \subset \Omega_{k}$ be a finite set of places such that the $G^{\sigma}$-torsor $g^{\sigma}: Z^{\sigma} \rightarrow X$ extends to a $\mathcal{G}_{\sigma}$-torsor $g^{\sigma}: \mathcal{Z}_{\sigma} \rightarrow \mathcal{X}$, where $\mathcal{G}_{\sigma}$ is a $\mathcal{O}_{k, S_{0}}$-group scheme and $\mathcal{Z}_{\sigma}$ and $\mathcal{X}$ are integral $\mathcal{O}_{k, S_{0}}$-schemes. For any finite subset $S^{\prime} \subset \Omega_{k}-S$, which we can enlarge without loss of generality so to contain $S_{0}-\left(S \cap S_{0}\right)$, let

$$
\prod_{v \in S^{\prime}} U_{v} \times \prod_{v \in \Omega_{k}-\left(S^{\prime} \cup S\right)} \mathcal{X}\left(\mathcal{O}_{v}\right) \subset X\left(\mathbf{A}_{k}^{S}\right)
$$

be a basic open neighbourhood of $\left(x_{v}\right)$, where the $U_{v} \subset X\left(k_{v}\right)$ are open with respect to the local topology. Since the torsor $g^{\sigma}: Z^{\sigma} \rightarrow X$ induces a continuous map $g^{\sigma}: Z^{\sigma}\left(k_{v}\right) \rightarrow X\left(k_{v}\right)$ with respect to the local topology, it follows, by continuity, that $\left(g^{\sigma}\right)^{-1}\left(U_{v}\right) \subset Z^{\sigma}\left(k_{v}\right)$ is also open. Hence, the basic open set in $Z^{\sigma}\left(\mathbf{A}_{k}^{S}\right)$ given by

$$
\prod_{v \in S^{\prime}}\left(g^{\sigma}\right)^{-1}\left(U_{v}\right) \times \prod_{v \in \Omega_{k}-\left(S^{\prime} \cup S\right)} \mathcal{Z}_{\sigma}\left(\mathcal{O}_{v}\right) \subset Z^{\sigma}\left(\mathbf{A}_{k}^{S}\right)
$$


contains $\left(z_{v}\right)$. Since by assumption $\left(z_{v}\right) \in{\overline{Z^{\sigma}(k)}}^{S}$, it follows that there exists some $z \in \operatorname{pr}^{S}\left(\delta\left(Z^{\sigma}(k)\right)\right) \cap$ $\prod_{v \in S^{\prime}}\left(g^{\sigma}\right)^{-1}\left(U_{v}\right) \times \prod_{v \in \Omega_{k}-\left(S^{\prime} \cup S\right)} \mathcal{Z}_{\sigma}\left(\mathcal{O}_{v}\right)$. Then $g^{\sigma}(z) \in \operatorname{pr}^{S}(\delta(X(k))) \cap \prod_{v \in S^{\prime}} U_{v} \times \prod_{v \in \Omega_{k}-\left(S^{\prime} \cup S\right)} \mathcal{X}\left(\mathcal{O}_{v}\right)$, and we are done.

Proof of Theorem 1.5. We have a natural $H$-torsor structure on $G \rightarrow G / H$. For any $\sigma \in H_{\text {ét }}^{1}(k, H)$, since $G^{\sigma}$ is a connected linear algebraic group over $k$, it follows by our hypotheses and by Dem11, Corollaire 3.20] that $G^{\sigma}$ satisfies strong approximation off $S$ with algebraic Brauer-Manin obstruction (and thus with étale-Brauer obstruction). Hence, Theorem 1.4 yields that $G / H$ satisfies strong approximation off $S$ with étale-Brauer obstruction.

Proof of Theorem 1.10. By classical results due to Kneser and Platonov (see e.g. PR94, Theorem 7.12]), under our assumptions we know that $G^{\sigma}$ satisfies strong approximation off $S$ for all $\sigma \in$ $H_{\text {ét }}^{1}(k, H)$. Let $\pi: G \rightarrow G / H$ be the natural $H$-torsor. Using [CDX19, Proposition 6.3], a similar proof as the one of [Sto07, Proposition 5.17] yields that

$$
(G / H)\left(\mathbf{A}_{k}\right)^{\text {ét }}=\bigcup_{\sigma \in H_{\text {ét }}^{1}(k, H)} \pi^{\sigma}\left(G^{\sigma}\left(\mathbf{A}_{k}\right)^{\text {ét }}\right) .
$$

Since $G^{\sigma}$ satisfies strong approximation off $S$, in particular $G^{\sigma}$ satisfies strong approximation with respect to the finite descent obstruction off $S$ (or we could just notice that, since $\pi_{1}^{\text {ét }}\left(G_{\bar{k}}^{\sigma}\right)=0$, we have $\left.G^{\sigma}\left(\mathbf{A}_{k}\right)^{\text {ét }}=G^{\sigma}\left(\mathbf{A}_{k}\right)\right)$. Hence, by arguing in a similar way as in the proof of Theorem 1.5 , we deduce that

$$
(G / H)\left(\mathbf{A}_{k}^{S}\right)^{\text {ét }} \subset \overline{(G / H)(k)}{ }^{S} .
$$

The fibration obtained by applying the analytification functor $(-)^{\text {an }}$ to the $H_{\mathbf{C}}$-torsor $G_{\mathbf{C}} \rightarrow$ $(G / H)_{\mathbf{C}}$ induces the homotopy (exact) sequence

$$
\pi_{1}^{\text {top }}\left(\left(G_{\mathbf{C}}\right)^{\text {an }}\right) \rightarrow \pi_{1}^{\text {top }}\left(\left((G / H)_{\mathbf{C}}\right)^{\text {an }}\right) \rightarrow \pi_{0}^{\text {top }}\left(\left(H_{\mathbf{C}}\right)^{\text {an }}\right) \rightarrow \pi_{0}^{\text {top }}\left(\left(G_{\mathbf{C}}\right)^{\text {an }}\right),
$$

where $\pi_{0}^{\text {top }}\left(\left(G_{\mathbf{C}}\right)^{\text {an }}\right)=0$ since $\left(G_{\mathbf{C}}\right)^{\text {an }}$ is (topologically) connected as $G_{\mathbf{C}}$ is (see Gro71, XII, Proposition 2.4]), and where $\pi_{1}^{\text {top }}\left(\left(G_{\mathbf{C}}\right)^{\text {an }}\right)=0$ since $\left(G_{\mathbf{C}}\right)^{\text {an }}$ is (topologically) simply connected. In order to see this last claim, note that by [Gro71, XII, Corollaire 5.2] we have (omitting base-points) that $\overline{\pi_{1}^{\text {top }}\left(\left(G_{\mathbf{C}}\right)^{\text {an }}\right)} \cong \pi_{1}^{\text {et }}\left(G_{\mathbf{C}}\right)$. Moreover, since $G$ is simply connected, we have $\pi_{1}^{\text {et }}\left(G_{\bar{k}}\right)=0$. By the Lefschetz principle for étale fundamental groups (see Sza09, Second proof of Corollary 5.7.6 and Remark 5.7.8] together with [Gro71, XII] and $\operatorname{Org} 03)$, this is equivalent to $\pi_{1}^{\text {ét }}\left(G_{\mathbf{C}}\right)=0$. But since $\pi_{1}^{\text {top }}\left(\left(G_{\mathbf{C}}\right)^{\text {an }}\right)$ is finitely generated and abelian, it follows that $\overline{\pi_{1}^{\text {top }}\left(\left(G_{\mathbf{C}}\right)^{\text {an }}\right)}=0$ implies that $\pi_{1}^{\text {top }}\left(\left(G_{\mathbf{C}}\right)^{\text {an }}\right)=0$, which proves the claim.

Since $H$ is finite, since $\left((G / H)_{\mathbf{C}}\right)^{\text {an }}$ is (topologically) connected as $(G / H)_{\mathbf{C}}$ is, and by the Lefschetz principle for étale fundamental groups, it follows that taking the profinite completion of $\pi_{1}^{\text {top }}\left(\left((G / H)_{\mathbf{C}}\right)^{\text {an }}\right)=\pi_{0}^{\text {top }}\left(\left(H_{\mathbf{C}}\right)^{\text {an }}\right)$ yields that

$$
\pi_{1}^{\text {ét }}\left((G / H)_{\bar{k}}\right)=\overline{\pi_{0}^{\text {top }}\left(\left(H_{\mathbf{C}}\right)^{\text {an }}\right)}
$$

is finite of size $\# H(\bar{k})$.

Since $(G / H)(k) \neq \emptyset$ and $(G / H)$ is geometrically connected (as it is connected and it has a $k$ rational point), by [Sto07, Lemma 5.7(1),(2)] (see also the errata in [Sto]) we have

$$
(G / H)\left(\mathbf{A}_{k}\right)^{\text {ét }}=\bigcap_{i \in I} \bigcup_{\sigma \in H_{\text {êt }}^{1}\left(k, F_{i}\right)} f_{i}^{\sigma}\left(Y_{i}^{\sigma}\left(\mathbf{A}_{k}\right)\right),
$$

where each $f_{i}: Y_{i} \rightarrow G / H$ is some $F_{i}$-torsor over $G / H$ under a finite linear algebraic group $F_{i}$ over $k$ with $F_{i}(\bar{k})$ a quotient of $H(\bar{k})$ (in particular, $\left.\# F_{i}(\bar{k}) \mid \# H(\bar{k})\right)$ and with $Y_{i}$ geometrically connected. By hypothesis, $H$ belongs to a $\left(k\right.$-isomorphism) class $\mathscr{F} \subset \mathcal{F}_{k}$ satisfying the following property: for 
all $F \in \mathcal{F}_{k}$, if $F(\bar{k})$ is the quotient of some $F^{\prime}(\bar{k})$ by some $F^{\prime \prime}(\bar{k})$ with $F^{\prime} \in \mathscr{F}$ and $F^{\prime \prime} \in \mathcal{F}_{\bar{k}}$, then $F \in \mathscr{F}$. It is clear then that $F_{i} \in \mathscr{F}$ for all $i \in I$. It follows that

$$
(G / H)\left(\mathbf{A}_{k}\right)^{\text {ét }} \subset(G / H)\left(\mathbf{A}_{k}\right)^{\mathscr{F}} \subset \bigcap_{i \in I} \bigcup_{\sigma \in H_{\text {êt }}^{1}\left(k, F_{i}\right)} f_{i}^{\sigma}\left(Y_{i}^{\sigma}\left(\mathbf{A}_{k}\right)\right)=(G / H)\left(\mathbf{A}_{k}\right)^{\text {ét }^{2}}
$$

and thus that $(G / H)\left(\mathbf{A}_{k}\right)^{\text {ét }}=(G / H)\left(\mathbf{A}_{k}\right)^{\mathscr{F}}$. Hence, by (2.1) the result follows.

\section{ACKNowledGements}

The author would like to thank Yang Cao for suggesting the application to homogeneous spaces, Julian Demeio for sharing his preprint Dem20] with her, and the anonymous referee for their comments and suggestions that greatly improved the paper. This project has received funding from the European Union's Horizon 2020 research and innovation programme under the Marie SkłodowskaCurie grant agreement 840684 .

\section{REFERENCES}

[BD13] M. Borovoi and C. Demarche, Manin obstruction to strong approximation for homogeneous spaces, Comment. Math. Helv. 88 (2013), no. 1, 1-54.

[Bor96] M. Borovoi, The Brauer-Manin obstructions for homogeneous spaces with connected or abelian stabilizer, J. Reine Angew. Math. 473 (1996), 181-194.

[Cao18] Y. Cao, Approximation forte pour les variétés avec une action d'un groupe linéaire, Compositio Mathematica 154 (2018), no. 4, 773-819.

[Cao20] $\_$Sous-groupe de brauer invariant et obstruction de descente itérée, 2020. To appear in Algebra \& Number Theory.

[CDX19] Y. Cao, C. Demarche, and F. Xu, Comparing descent obstruction and Brauer-Manin obstruction for open varieties, Trans. Amer. Math. Soc. 371 (2019), 8625-8650.

[CX18a] Y. Cao and F. Xu, Strong approximation with Brauer-Manin obstruction for groupic varieties, Proc. London Math. Soc. 117 (2018), no. 3, 727-750.

[CX18b] _ Strong approximation with Brauer-Manin obstruction for toric varieties, Annales de l'Institut Fourier (Grenoble) 68 (2018), no. 5, 1879-1908.

[Dem11] C. Demarche, Le défaut d'approximation forte dans les groupes linéaires connexes, Proc. London Math. Soc. 102 (2011), no. 3, 563-597.

[Dem17] _ Obstructions de Brauer-Manin entières sur les espaces homogènes à stabilisateurs finis nilpotents, Bulletin de la SMF 145 (2017), no. 2, 225-236.

[Dem20] J. L. Demeio, The étale Brauer-Manin obstruction to strong approximation on homogeneous spaces (2020). Preprint.

[dJ] A. J. de Jong, A result of Gabber. Available at http://www . math.columbia.edu/ dejong/papers/2-gabber.pdf.

[Eke90] T. Ekedahl, An effective version of Hilbert's irreducibility theorem, Séminaire de Théorie des Nombres, Paris 1988-1989, Progr. Math., vol. 91, Birkhäuser Boston, 1990.

[Gro71] A. Grothendieck, Revêtements étales et groupe fondamental (SGA 1), Lecture notes in mathematics, vol. 224, Springer-Verlag, 1971.

[Har02] D. Harari, Groupes algébriques et points rationnels, Math. Ann. 322 (2002), no. 4, 811-826.

[Har07] _ Q Quelques propriétés d'approximation reliées à la cohomologie galoisienne d'un groupe algébrique fini, Bull. Soc. Math. France 135 (2007), no. 4, 549-564.

[HW18] Y. Harpaz and O. Wittenberg, Zéro-cycles sur les espaces homogènes et problème de Galois inverse, 2018. arXiv:1802.09605.

[Org03] F. Orgogozo, Altérations et groupe fondamental premier à p, Bulletin de la société mathématique de France 131 (2003), no. 1, 123-147.

[PR94] V. Platonov and A. Rapinchuk, Algebraic groups and number theory, Pure and Applied Mathematics, vol. 139, Academic Press Inc., Boston, MA, 1994.

[Ser08] J.-P. Serre, Topics in Galois theory (2nd ed.), Research Notes in Mathematics, vol. 1, A K Peters, 2008.

[Sko01] A. N. Skorobogatov, Torsors and rational points, Cambridge Tracts in Mathematics, vol. 144, Cambridge University Press, 2001.

[Sto07] M. Stoll, Finite descent obstructions and rational points on curves, Algebra \& Number Theory 1 (2007), no. 4, 349-391.

[Sto] _ Errata corrige to "Finite descent obstructions and rational points on curves". Available at http://www.mathe2. uni-bayreuth.de/stoll/papers/Errata-FiniteDescent.pdf. 
[Sza09] T. Szamuely, Galois groups and fundamental groups, Cambridge Studies in Advanced Mathematics, Cambridge University Press, 2009.

IST Austria, Am Campus 1, 3400 Klosterneuburg, Austria

Email address: fbalestr@ist.ac.at

$U R L:$ https://fbalestrieri.github.io 